\title{
IL-17-Induced Pulmonary Pathogenesis during Respiratory Viral Infection and Exacerbation of Allergic Disease
}

\author{
Sumanta Mukherjee, ${ }^{\star}$ Dennis M. Lindell, ${ }^{\dagger}$ \\ Aaron A. Berlin, ${ }^{*}$ Susan B. Morris, ${ }^{*}$ \\ Thomas P. Shanley, ${ }^{\ddagger}$ Marc B. Hershenson, ${ }^{\ddagger}$ and \\ Nicholas W. Lukacs* \\ From the Departments of Patbology, ${ }^{*}$ and Pediatrics and \\ Communicable Diseases, ${ }^{\ddagger}$ University of Michigan Medical School, \\ Ann Arbor, Michigan; and the Seattle Childrens Research \\ Institute, ${ }^{\dagger}$ Seattle, Washington
}

Severe respiratory syncytial virus (RSV) infections are characterized by airway epithelial cell damage, mucus hypersecretion, and Th2 cytokine production. Less is known about the role of $\mathrm{IL}-17$. We observed increased IL- 6 and IL-17 levels in tracheal aspirate samples from severely ill infants with RSV infection. In a mouse model of RSV infection, timedependent increases in pulmonary IL-6, IL-23, and IL-17 expression were observed. Neutralization of IL-17 during infection and observations from IL$17^{-/-}$knockout mice resulted in significant inhibition of mucus production during RSV infection. RSV-infected animals treated with anti-IL-17 had reduced inflammation and decreased viral load, compared with control antibody-treated mice. Blocking IL-17 during infection resulted in significantly increased RSV-specific CD8 T cells. Factors associated with CD8 cytotoxic T lymphocytes, T-bet, IFN- $\gamma$, eomesodermin, and granzyme $B$ were significantly up-regulated after IL-17 blockade. Additionally, in vitro analyses suggest that IL-17 directly inhibits T-bet, eomesodermin, and IFN- $\gamma$ in $\mathrm{CD8} \mathrm{T}$ cells. The role of IL-17 was also investigated in $\mathrm{RSV}$-induced exacerbation of allergic airway responses, in which neutralization of $\mathrm{IL}-17$ led to a significant decrease in the exacerbated disease, including reduced mucus production and Th2 cytokines, with decreased viral proteins. Taken together, our data demonstrate that IL-17 plays a pathogenic role during RSV infections. (Am J Pathol 2011, 179:248-258; DOI: 10.1016/j.ajpath.2011.03.003)
Nearly $98 \%$ of all infants become infected with respiratory syncytial virus (RSV) by the age of 2 years and experience severe bronchiolitis because their small airways easily become occluded. ${ }^{1}$ It is estimated by the U.S. Centers for Disease Control that up to 125,000 pediatric hospitalizations in the United States each year are due to RSV. In addition, RSV is pathogenic for elderly patients and for those with chronic lung disease and asthma, and further is associated with a mortality rate of $30 \%$ to $100 \%$ in immunosuppressed individuals. ${ }^{2,3} \mathrm{RSV}$ also is associated with acute exacerbations of chronic obstructive pulmonary disease, causing prolonged episodes of illness. Recurrent infections with RSV are common, and the pulmonary pathology is known to persist long after the virus has been cleared efficiently. RSV disease pathology is clinically characterized by airway hyperreactivity (AHR), increased mucus production, and inflammation. ${ }^{4-6}$ An altered immune environment due to an imbalance in the CD4 helper Th1 and Th2 responses is thought to underlie this disease phenotype. Recently it was reported that IL-17, produced by a subset of CD4 helper T cells (Th17 cells), was regulated by STAT-1 during RSV infections in rodents. ${ }^{7}$ The exact role of IL-17 in RSV disease pathogenicity is not known.

Interleukin-17 belongs to a family of cytokines that has six members: IL-17 (also called IL-17A, the prototype) and IL-17B through IL-17F; IL-17E is also known as IL-25. $\mathrm{IL}-17 \mathrm{~F}$ shares the strongest homology to $\mathrm{IL}-17 .^{8,9}$ Both IL-17 and IL-17F are proinflammatory and have overlapping roles in the development of various autoimmune disorders, such as rheumatoid arthritis, multiple sclerosis, and inflammatory bowel disease. However, IL-17 plays a critical role in host defense during bacterial and fungal infection, whereas IL-17F is largely involved in the development of asthma and airway inflammation. ${ }^{9-11}$ Moreover. IL-17F does not up-regulate proinflammatory molecules to the same degree as does $\mathrm{IL}-17 .{ }^{12}$ The re-

\footnotetext{
Supported by the NIH (Al-073876 and Al-036302 to N.W.L.).

Accepted for publication March 17, 2011.

Address reprint requests to Nicholas W. Lukacs, Ph.D., University of Michigan Medical School, Department of Pathology, Ann Arbor, MI 48109. E-mail: nlukacs@umich.edu.
} 
ceptors for IL-17 and IL-17F are IL-17RA and IL-17RC, respectively; because these receptors have different tissue expression, the isoforms of IL-17 are tissue-specific. Expression of IL-17RC is limited to nonhematopoietic cells, but IL-17RA is expressed ubiquitously. IL-17 may promote Th2 responses in the lung through IL-17RA, whereas IL-17F has a regulatory role in limiting allergic asthma development. ${ }^{12-14}$

The combination of AHR and mucus production in the airways is a significant clinical outcome during viral infections. RSV infections induce significant AHR and mucus production in the airways of mice ${ }^{7,15,16}$ and induce neutrophilia in the lung epithelium. ${ }^{11,12,17}$ Although IL-17 has been reported to play a pathogenic role during the development of asthma by regulating mucin gene expression in the airways, ${ }^{11}$ its specific role in pathogenic responses during RSV infection is not known.

Here, we report increased IL-17 production in infants with RSV infection and identify a role of IL-17 in a mouse model of primary RSV infection, as well as during viral exacerbation of allergic lung disease. Using either IL-17deficient mice or neutralization of IL-17 significantly inhibited mucus production during RSV infection. In addition, blocking IL-17 significantly decreased viral load and altered cytotoxic CD8 T-cell marker expression. These responses were also observed in RSV-induced allergic airway exacerbation, suggesting that IL-17 plays an important role in the pathogenesis of RSV-induced disease.

\section{Materials and Methods}

\section{Mice}

Female BALB/c mice, 6 to 8 weeks old, were purchased from the Jackson Laboratory (Bar Harbor, ME). The IL$17^{-1-}$ mice, derived from breeder animals from the Jackson Laboratory, were a kind gift of Dr. Kathryn Eaton (University of Michigan). ${ }^{18}$ All mice were maintained in specific-pathogen-free facilities in the Unit for Laboratory Animal Medicine at the University of Michigan. The University Committee of Use and Care of Animals (UCUCA), University of Michigan, Ann Arbor, approved all animal experimental protocols, and experiments were conducted according to the guidelines provided by the UCUCA review committee.

\section{Human Specimens}

All human studies were performed in accordance with an approved University of Michigan institutional review board protocol after legal consent. The tracheal aspirate samples were diluted 50:50 with PBS containing complete anti-protease cocktail (Sigma-Aldrich, St. Louis, $\mathrm{MO}$ ) and $0.5 \%$ Triton $\mathrm{X}-100$ nonionic detergent to dissociate the mucus. Samples were aliquoted in $75 \mu \mathrm{L}$ and stored at $-80^{\circ} \mathrm{C}$ until analysis. IL-17 was analyzed using a Bio-Plex 200 System (Bio-Rad Laboratories, Hercules, CA) and IL-6 was analyzed using an enzyme-linked immunosorbent assay (human Quantikine ELISA kit; R\&D Systems, Minneapolis, MN). Tracheal aspirate samples were obtained from RSV-infected infants who were hospitalized and given mechanical ventilation in a pediatric intensive care unit. Control uninfected tracheal infant samples were obtained from children undergoing surgery for reasons unrelated to RSV infection, after parental consent.

\section{RSV Infection and Cockroach Sensitization}

Female BALB/c mice, 6 to 8 weeks old, were anesthetized and infected with RSV on day 0 . Lungs were harvested on days $1,2,4,6,8,10$, and 12 for the time-course studies and on day 8 for all other studies. Cockroach (CRA) sensitization was performed as described previously. ${ }^{19,20}$ Briefly, mice were sensitized with a 1:1 mixture of clinical-grade CRA extract ( $\mathrm{Hol}-$ lister-Stier Laboratories, Spokane, WA) and incomplete Freund's adjuvant (Sigma-Aldrich), administered subcutaneously and intraperitoneally on day 0 . This cockroach allergen is a skin test/immunotherapy-grade preparation that has very little endotoxin contamination $(<10 \mathrm{ng} / \mathrm{mL})$. At day 14 , mice were sensitized by intranasal challenges of CRA, which was followed by RSV infection on day 16. In animals treated with antibodies, control or anti-IL-17 purified IgG was given 2 hours before RSV infection on day 16. Two doses of control or anti-IL-17 purified IgG were also given on days 18 and 20. After a final intranasal challenge of CRA on day 21, the mice were euthanized and AHR studies were conducted on day 22. The lungs and draining lymph nodes (DLNs) of these mice were isolated for further characterization.

\section{RSV and Viral Plaque Assay}

RSV A strain (line 19) was derived from a clinical isolate at the University of Michigan. ${ }^{21,22}$ The virus was administered to mice intratracheally by tongue pull at $1 \times 10^{5}$ plaque-forming units (PFU). ${ }^{23-25}$ We have previously shown that the pathophysiology associated with UV-inactivated virus is comparable to that of naïve mice without RSV infection, ${ }^{26-28}$ and in the present study UVinactivated virus failed to induce IL-17 in mice (data not shown). Plaque assays were performed on RSV-infected lungs. Whole lungs were harvested and ground with sand using a mortar and pestle. Supernatants were serially diluted and incubated with Vero cells for 4 days. Viral plaques were determined using a RSV-specific polyclonal antibody (Millipore, Billerica, MA).

\section{Generation of Rabbit Anti-Mouse Polyclonal IL-17-Specific Antibody}

Rabbit anti-mouse IL-17 antibodies were prepared by multiple-site immunization of New Zealand White rabbits with recombinant mouse IL-17 (R\&D Systems) in complete Freund's adjuvant and boosted with IL-17 in incomplete Freund's adjuvant, according to procedures from our laboratory as described previously (both purchased from Sigma-Aldrich). ${ }^{29}$ Polyclonal antibodies were titered by direct ELISA against IL-17 coated onto 96-well plates. 
Specificity of the antibody was tested by direct ELISA against IL-17, IL-17E, and IL-17F, as described previously, ${ }^{29-31}$ and was found to react only with IL-17. The polyclonal antibody was further purified from the serum using Protein A columns (Pierce; Thermo Fisher Scientific, Rockford, IL) according to the manufacturer's protocol. Control antibody (Cab) was purified from serum of unimmunized mice also using Protein A columns.

\section{Neutralization of IL-17}

Mice were pretreated intraperitoneally with $2.5 \mathrm{mg}$ of purified polyclonal anti-mouse IL-17 antibody 2.5 hours before RSV infection on day 0 and then every other day until day 6 . The control group similarly received $2.5 \mathrm{mg}$ of anti-mouse IgG antibody (Cab).

\section{Airway Response}

Airway hyperreactivity was assessed as described previously. ${ }^{19,32-34}$ Briefly, mice were anesthetized with sodium pentobarbital, intubated via cannulation of the trachea, and ventilated with a Harvard pump ventilator ( 0.3 $\mathrm{mL}$ tidal volume; 120 breaths/minute). Airway hyperreactivity was measured using a direct ventilation methodology with a sensitive mouse plethysmograph and software for calculation of the measurements (Buxco Research Systems, Wilmington, NC). After baseline measurements, mice were injected intravenously with $7.5 \mu \mathrm{g}$ of methacholine (Sigma-Aldrich), and the peak airway resistance was recorded as a measure of AHR.

\section{Determination of RSV Antigens in Lungs of Infected Mice}

Total RSV antigen levels in lung homogenates were measured by a specific ELISA as described previously. 35,36 Briefly, the lung samples $(100 \mu \mathrm{L})$ were incubated for 1 hour in 96-well plates coated with goat anti-RSV antibody (Millipore). After three washes, the plates were incubated with a mouse anti-RSV polyclonal secondary antibody (Vector Laboratories, Burlingame, CA). After a final incubation with a peroxidase-labeled goat anti-mouse IgG antibody (Millipore), positive wells were assessed by 0 phenylenediamine dihydrochloride (Dako, Carpinteria, CA) substrate development. Optical density readings were at $450 \mathrm{~nm}$.

\section{Histology and RT-PCR}

For histology studies, right-lung lobes from infected mice were removed, fixed in 10\% formalin, and stained with H\&E or PAS to detect mucus production. For RT-PCR, the lower left lobe of the freshly harvested lung was snap-frozen and stored at $-80^{\circ} \mathrm{C}$. Total RNA was extracted from the frozen tissue using TRIzol reagent (Invitrogen, Carlsbad, CA) and was reverse transcribed to CDNA. Real-time PCR was performed with the cDNA using gene-specific primers. Murine primers for IL-4, IL-5, IL-13, IFN- $\gamma$, IL-17, IL-17F, IL-23p19, IL-6, RANTES, KC, eotaxin, MDC, MIP1a, MIP2a, T-bet, eomesodermin (Eomes), and GAPDH were purchased from Applied Biosystems (Carlsbad, CA). Primers and probes for Muc5ac, Gob5, RSV-F, RSV-N, and RSV-G were determined using primer/probe detection sets designed by PE Biosystems (Foster City, CA) and purchased from SigmaAldrich. All results were normalized to GAPDH expression.

\section{Protein Assays}

Single-cell suspensions of lymph nodes were seeded at a concentration of $5 \times 10^{6}$ cells $/ \mathrm{mL}$ in a 96-well plate and were restimulated with RSV. At 48 hours, the supernatants were harvested and analyzed for cytokines, which were quantified using a Bio-Plex bead-based (Luminex) cytokine assay purchased from Bio-Rad Laboratories.

\section{Flow Cytometry}

Single-cell suspensions of lung (after collagenase dispersion) and lymph node cells were prepared as described previously. ${ }^{37}$ To detect various surface markers (CD3, CD8, CD4, and CD69 from eBioscience, San Diego, CA; pan NK and $\gamma \delta$ T cell from BioLegend, San Diego, CA; and IL-17RA from R\&D Systems), cells were stained with the indicated antibodies after 10 minutes of preincubation with Fc block (BD Biosciences, San Jose, CA). For detection of RSV M protein-specific T-cell receptor-positive CD8 T cells (RSV tetramer, $\mathrm{M}_{82-90} ; \mathrm{H} 2-\mathrm{k}^{\mathrm{d}}$ ), staining antibody was added 30 minutes before the addition of any other antibody and the reaction took place at room temperature. The cells were fixed overnight with $4 \%$ formalin. For intracellular staining, the cells were fixed and permeabilized using a staining buffer kit (eBioscience) and then stained with antibodies against T-bet, granzyme B, IFN- $\gamma$, and Eomes (all from eBioscience). The samples were processed through a flow cytometer (LSRII; Becton-Dickinson, Franklin Lakes, NJ). The data were analyzed using FlowJo software version 8.8.4 (TreeStar, Ashland, OR).

\section{In Vitro CD8 T-Cell Culture}

CD8 T cells were sorted from the spleen using magnetic beads and plated in 96-well plates at a concentration of $2 \times 10^{5}$ cells per well in RPMI-1640 complete medium (Lonza, Walkersville, MD). The cells were stimulated with plate-coated anti-CD3 and soluble anti-CD28 (eBioscience) with or without recombinant IL-17 (R\&D Systems) at $100 \mathrm{ng} / \mathrm{mL}$. RNA was extracted from the cells at 6 hours after stimulation and was quantified by real-time RT-PCR.

\section{Bronchoalveolar Lavage Fluid Microscopy}

After AHR measurement and cervical dislocation, bronchoalveolar lavage fluid was collected from each mouse. One milliliter sterile $0.9 \mathrm{~N}$ saline was instilled intratracheally and was suctioned out after a few seconds. Samples were placed in Eppendorf tubes and were centrifuged at $1500 \mathrm{rpm}$ for 5 minutes $(240 \times \mathrm{g})$. The cells were centrifuged in a shandon cytospin 2, fixed, and a differential 
cell count was done after Diff-Quik staining (Dade Behring, Newark, DE).

\section{Statistical Analysis}

Data are reported as means \pm SE. Statistical significance was determined by Student's t-test or one-way analysis of variance with Newman-Keuls post hoc test. $P<0.05$ was considered significant.

\section{Results}

\section{RSV Infection Induces IL-17 Production in Mice and Humans}

To investigate whether RSV infection induces IL-17 production in vivo, BALB/c mice were infected with RSV and a time-course analysis was performed of mRNA expression of two different IL-17 transcripts (IL-17 and IL-17F) and the cytokines involved in their production (IL-6) and maintenance (IL-23p19). RSV infection significantly induced IL-17 but not IL-17F in the lungs of infected mice compared with control mice, with expression levels peaking at day 8 after infection (Figure 1A). IL-6 and IL-23p19 mRNA expression increased threefold and twofold, respectively, on day 8 after RSV infection (Figure 1A). RSV infection also increased IL-17 protein production in the lungs, and peak IL-17 levels correlated with the transcript levels (Figure 1B). Furthermore, we observed a significant increase in antigen-specific IL-17 production in the DLNs taken at day 8 from infected animals when they were restimulated ex vivo with RSV (Figure $1 \mathrm{C}$ ).

To determine which lung cells were expressing IL-17 during peak IL-17 production, CD4 T cells, pan natural killer (NK) cells, and $\gamma \delta$ T cells taken at day 8 from RSV-infected lungs were sorted by flow cytometry and intracellular IL-17 levels were measured. We observed significant increase in $\mathrm{IL}-17^{+} \mathrm{CD} 4 \mathrm{~T}$ cells in the lungs of RSV-infected mice, compared with uninfected mice (Figure 1D). For NK and $\gamma \delta \mathrm{T}$ cells, however, no significant difference was observed between infected and uninfected groups. These data demonstrate that RSV infection induces IL-17 expression by CD4 T cells in the lungs during peak IL-17 production. To validate our data, we further analyzed IL-6 and IL-17 levels in tracheal aspirate samples from infants who had been hospitalized and given mechanical ventilation because of RSV infection. Both cytokines were significantly increased in infected samples (Figure 1, E and F). Thus, IL-17 appears to be induced during RSV infection in severely ill infants and correspondingly in our animal model of RSV-induced disease.

\section{Neutralization of IL-17 Regulates Mucus Production and Neutrophilic Inflammation and Increases Viral Load in the Lung}

We next investigated the contribution of IL-17 to the increased pathogenesis in our mouse model of primary RSV infection. Mice were infected with RSV and treated
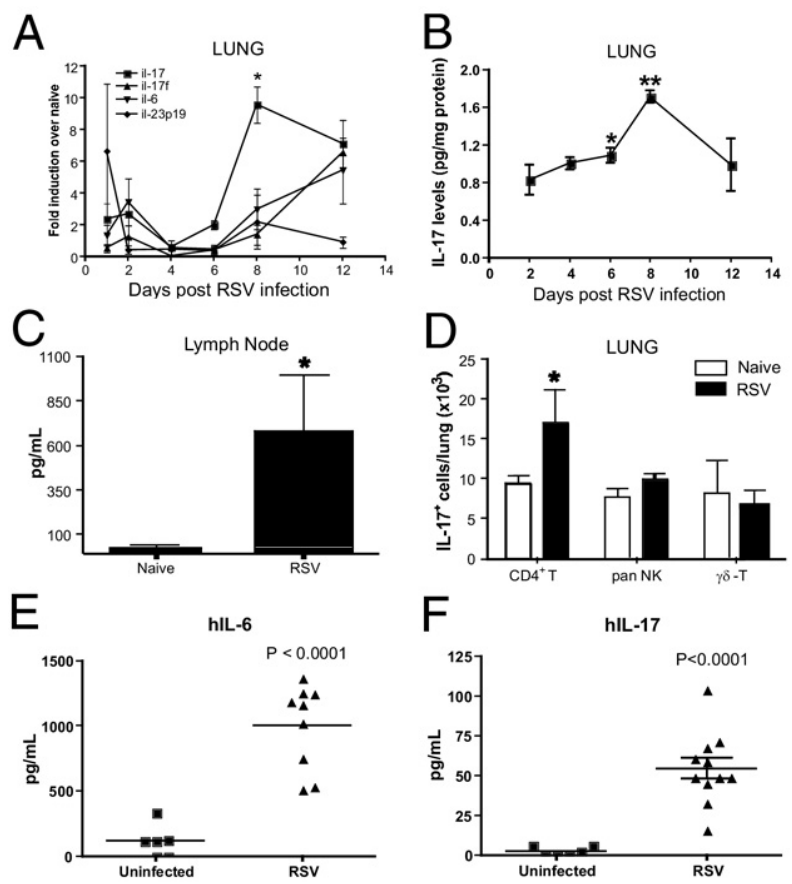

Figure 1. RSV infection induces IL-17 production in mice and humans, and CD4 T cells contribute significantly to peak IL-17 production. A: Total RNA was extracted from the lungs of RSV-infected BALB/c mice on days 1, 2, 4, 6, 8 , and 12 after infection, and the relative expression of IL-17, IL-17F, IL-6, and IL-23p19 was analyzed by real-time PCR. The expression levels were normalized to the housekeeping gene GAPDH and fold inductions were compared with naïve mice. B: IL-17 production in the lungs on days 2, 4, 6, 8, and 12 after RSV infection. The data were analyzed by a Bio-Plex system. C: Draining lymph nodes from naïve and RSV-infected mice were harvested on day 8 after infection and were rechallenged with RSV. Supernatants were collected 48 hours later, and IL-17 production was analyzed by a Bio-Plex system. D: Intracellular IL-17 levels were measured by flow cytometry in different IL-17 producing cells in the lungs of mice at day 8 after RSV infection and were compared with naïve lungs. Each experiment was repeated at least twice, with four to five mice per group. Human IL-6 (E) and IL-17 (F) were measured in tracheal aspirate samples from infants with or without RSV infection. Data are reported as means \pm SE. ${ }^{*} P<0.04,{ }^{* * *} P=0.036$.

intraperitoneally with anti-IL-17 or Cab every other day (Figure 2A). On day 8 after infection, we measured AHR and mucus production. Although we observed no change in AHR with anti-IL-17 antibody, compared with the Cab group (Figure 2B), neutralization of IL-17 significantly down-regulated the mucus-associated genes Muc5ac and Gob5 (Figure 2C). The decrease in the mucus gene expression was also reflected in the lung histology, with marked reduction of mucus and goblet cell staining in the large airways after neutralization of IL-17 (Figure 2D). Correspondingly, RSV infection of the IL$17^{-1-}$ knockout mice (on a C57BL6 background) further confirmed our observations with IL-17 neutralization. Absence of IL-17 in the knockout mice, even in the relatively resistant C57BL6 background mice, significantly downregulated mucus genes, a finding that was also reflected in the lung histology, with marked reduction in mucus staining (Figure 2, E and F).

Previous reports of increased neutrophil migration to the lung during RSV infections ${ }^{38-40}$ prompted us to investigate the role of IL-17 in neutrophilic infiltration. Although we observed no significant differences in eosinophil, macrophage, or lymphocyte numbers, there was a 


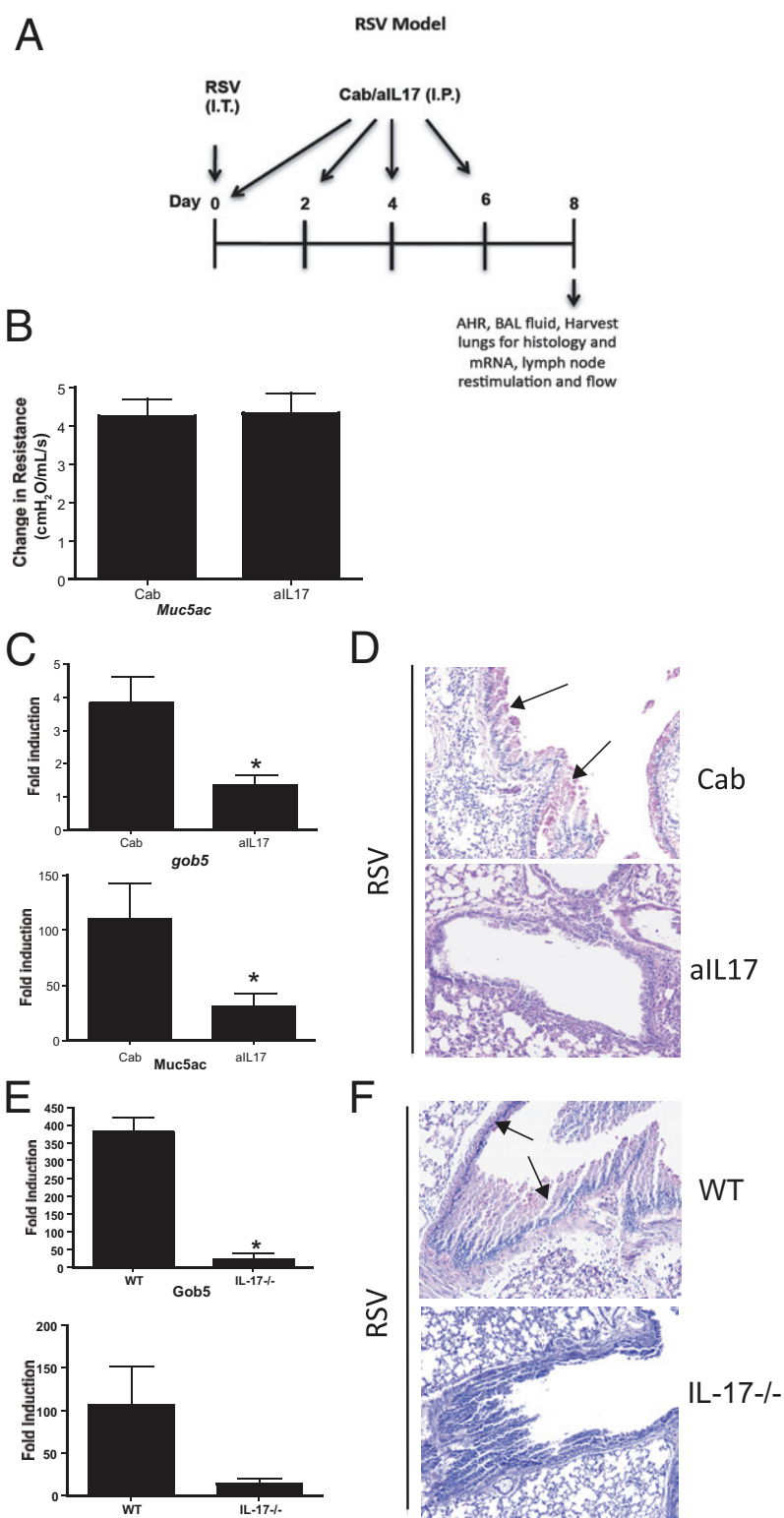

Figure 2. Neutralization of IL-17 inhibits mucus-associated genes and mucus accumulation in the lungs after RSV infection. A: Schematic representation of the primary RSV infection model. B: Airway resistance was measured at 8 days after RSV infection in mice treated with control or anti-IL-17 antibody (aIL17) after a single dose of methacholine. Data are represented as mean AHR in $\mathrm{cmH}_{2} \mathrm{O} / \mathrm{mL}$ per second $\pm \mathrm{SE}$. Relative expression of mucus-associated genes in the lungs of mice treated with Cab versus anti-IL-17 antibody (C) or of WT versus IL-1 $7^{-/-}$mice (E) was analyzed by real-time PCR. Data are reported as means $\pm \mathrm{SE}$. ${ }^{*} P<0.01$. Lungs from Cab versus alL17-treated mice (D) or WT versus IL-17 $7^{-/-}$mice (F) were harvested and stained with PAS. Arrows indicate PAS positive staining. Original magnification, $\times 200$. Each experiment was repeated three times, with five mice in each group.

significant decrease in the number of neutrophils in the bronchoalveolar lavage fluid from mice treated with antiIL-17 antibody, compared with the Cab-treated mice (Figure 3A). The decrease in neutrophils in the anti-IL-17 antibody-treated animals correlated with the decreased expression of the neutrophil-associated chemokine CXCL1 (mouse GRO- $\alpha$ ) in the lungs of RSV-infected mice (Figure 3B). Although the extent of the decrease was not great $(5 \%$ to $2 \%)$, increased neutrophilic inflammation may be a component of enhanced pathological responses to virus infection associated with IL-17.

An important aspect of infectious disease-associated pathology is the persistence (viral load) and/or clearance of virus. Therefore, we next investigated the kinetics of viral persistence after RSV infection in mice treated with Cab or anti-IL-17 by analyzing the changes in viral mRNA expression of the RSV nuclear protein (RSV-N). Blockade of IL-17 decreased RSV-N with time, with levels significantly lower on days 8 and 10 after infection, compared with Cab-treated mice (Figure 4A). The decrease in RSV-N expression in anti-IL-17 antibody-treated mice started as early as day 4 after infection, and to further confirm this finding we measured the viral load by assessing plaque-forming units from lungs of the virus RSVinfected mice. We found a marked decrease in viral titers in anti-IL-17 antibody-treated animals, compared with those given Cab (Figure 4B). Furthermore, given that we detected significantly lower levels of RSV-N on day 8 after infection, we next examined RSV-specific antigen levels in the lungs by ELISA on day 8 after infection, the time of peak IL-17 production. Anti-IL-17 antibodytreated animals demonstrated significantly lower levels of viral antigen in their lungs (used as one measure of viral clearance as described previously ${ }^{34}$ ) (Figure 4C). The results confirmed our RSV-N data and suggest that neutralization of IL-17 lowers viral load at 4 and 8 days after infection.

Although viral load is a critical determinant in virusinduced pathology, the inflammation observed during the disease progression is also associated with altered helper $T$ cell-induced cytokine environment. To address

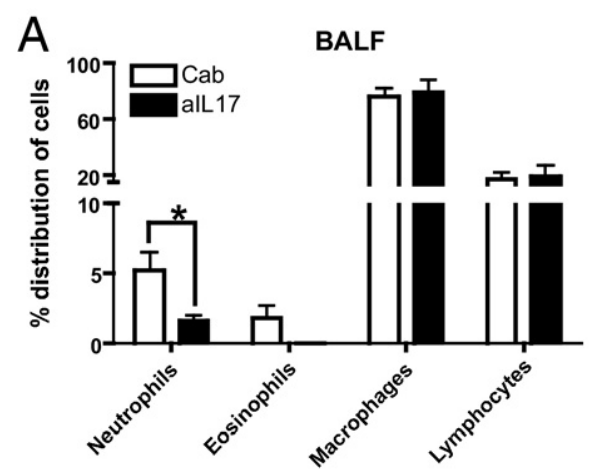

$\mathrm{B}$

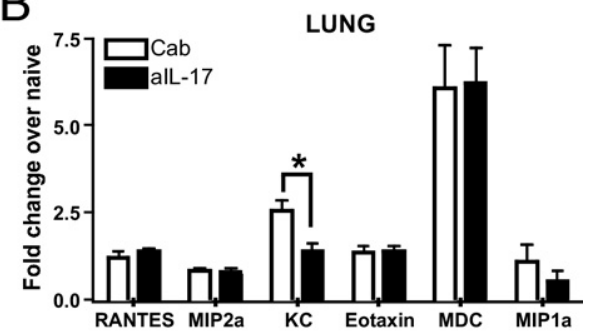

Figure 3. Blockade of IL-17 inhibits neutrophilic infiltration in bronchoalveolar lavage fluid (BALF). A: Differential count of BALF cellularity for RSV-infected mice treated with anti-IL-17 antibody (aIL17) or Cab. B: Relative expression of chemokines in the lungs analyzed by real-time PCR from RSV-infected mice treated with anti-IL-17 antibody (alL-17) or Cab. The experiment was repeated twice with five mice in each group. Data are reported as means \pm SE. ${ }^{*} P<0.05$. 
A
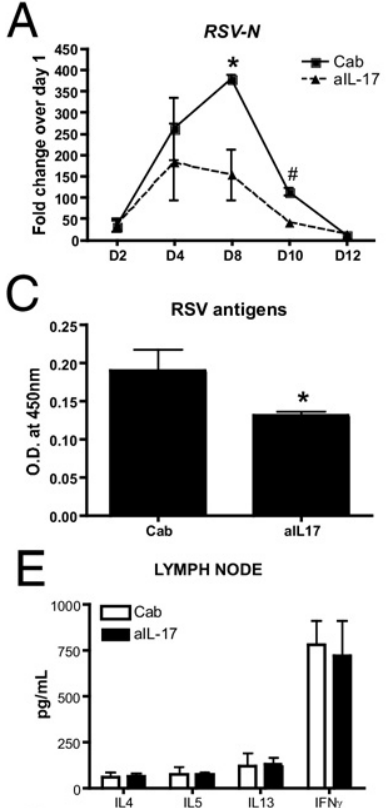

B

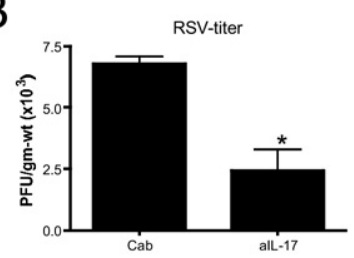

D

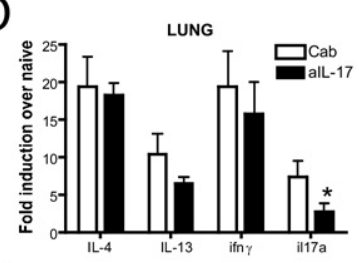

$\mathrm{F}$

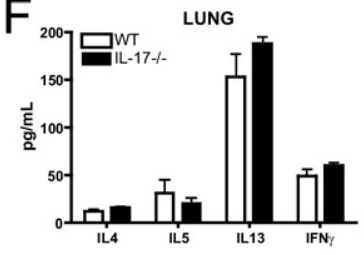

$\mathrm{G}$

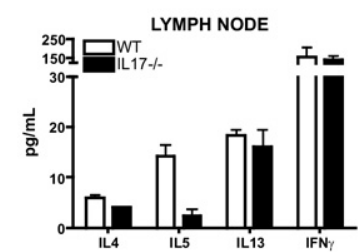

Figure 4. RSV-induced IL-17 reduces viral clearance but does not alter Th2 cytokines. A: RSV nuclear protein N expression in the lungs was measured by real-time PCR on days $2,4,8,10$, and 12 after RSV infection and treatment with Cab or anti-IL-17 antibody (aIL-17). The relative expression of RSV-N in $\mathrm{Cab}$ and aIL-17 treated groups was normalized to RSV-infected mice at day 1. B: Viral plaque-forming units (PFU) per wet weight of lung were measured 4 days after RSV infection and after treatment with anti-IL-17 antibody or Cab. Data are reported as means \pm SE. ${ }^{*} P<0.05$. The experiment was repeated twice with similar results. C: RSV antigens were measured by ELISA. O.D., optical density. D: Relative expression of cytokines IL-4, IL-13, IFN- $\gamma$, and IL-17A was analyzed by real-time PCR in the lungs of RSV-infected mice treated with Cab or aIL-17 on day 8 after infection. Cytokine mRNA expression levels were normalized to those of naïve mice. E: Lung DLNs from the same mice were restimulated with RSV for 48 hours and the supernatants were analyzed for cytokine production by a Bio-Plex system. Cytokine responses to RSV in WT and $\mathrm{IL}-17^{-/-}$mice were compared in the lungs $(\mathbf{F})$ and lymph nodes $(\mathbf{G})$. The experiment was repeated twice, with four to five mice per group. Data are reported as means $\pm \mathrm{SE} .{ }^{*} P<0.05$

this aspect, we examined the role of $\mathrm{IL}-17$ in specifically altering the immune cell responses. BALB/c mice were treated with anti-IL-17 antibody or Cab (intraperitoneally) 2.5 hours before RSV infection. At 8 days after infection, we assessed the cytokine expression in whole lungs by quantitative PCR and rechallenged the DLNs ex vivo with RSV for an additional 48 hours before analyzing antigen-specific Th1 and Th2 cytokine production. Neutralization of IL-17 significantly reduced the expression of IL-17 mRNA in the lungs (Figure 4D), but did not alter Th1 or Th2 cytokine responses. Similarly, the levels of the CD4 effector cytokines remained unaltered in the DLNs (Figure 4E). In IL-17 ${ }^{-1-}$ mice, no significant alteration of the Th2 cytokines was observed in either the lungs or the lymph nodes, compared with wild-type mice (Figure 4, F and G). Altogether, the data suggest that IL-17 has a limited role in altering the production of other key cytokines during primary RSV infection.

\section{IL-17 Alters CD8 T-Cell Response and Cytotoxic Function in the Lung during RSV Infection}

Because our studies suggested an alteration in viral clearance and an increase in CD4 T cells producing IL-17, we next investigated the lymphocyte compartment. We analyzed total CD4 T cell and CD8 T cell, as well as activated CD4 T cell (CD4CD69) and CD8 T cell (CD8CD69) populations, in both DLNs and lungs. Although there was no significant change in the total and activated CD4 and CD8 T-cell population in the DLNs of mice treated with anti-IL-17, compared with the Cab treatment (Figure 5A), there was a significant difference in both CD4 and CD8 T -cell numbers in the lungs (Figure 5B). Given the increased viral load in the presence of $\mathrm{IL}-17$, we next examined the expression of IL-17 receptor (IL-17RA) on CD8 T cells in the lungs of RSV-infected mice and found that RSV infection significantly increased IL-17RA expression on CD8 T cells (Figure $5 \mathrm{C}$ ). To further investigate the role of $\mathrm{IL}-17$ in RSV-specific responses of CD8 T cells in the lungs during viral infections, we assayed CD8 T cells expressing the RSV-specific immunodominant T-cell receptor using tetramer-specific staining in mice treated with Cab or neutralizing antibody to IL-17. There was a significant increase in immunodominant RSV-specific tetramer positive CD8 $\mathrm{T}$ cells after neutralization of $\mathrm{IL}-17$, both as percentage of $\mathrm{CD}^{+}$cells and total cells in the lung, suggesting that $\mathrm{IL}-17$ is critical in mounting a potent antiviral response (Figure 5, D and E).

Functional activation of cytotoxic $T$ lymphocytes (CTLs) can be assessed using a number of associated markers, including IFN- $\gamma$ production and its related transcription factors T-bet and Eomes. In addition, granzyme B can be used as a marker for CTL activation. Our data indicate that there was a significant increase in the IFN- $\gamma$ positive CD8 T cells (Figure 5F), as well as a significant increase of both Eomes (Figure $5 \mathrm{G}$ ) and T-bet (Figure $5 \mathrm{H}$ ) levels, in the lungs of mice treated with anti-IL-17 antibody, compared with Cab. Mice treated with anti-IL-17 antibody had significantly higher levels of granzyme B, compared with the Cabtreated mice (Figure $5 \mathrm{I}$ ), indicating that, in addition to regulating the IFN- $\gamma$ production by CD8 T cells, IL-17 also decreases cytotoxic $\mathrm{T}$ cell development during RSV infections, which in turn could inhibit proper viral clearance. To confirm that alteration of CD8 $\mathrm{T}$ cell activation is a direct effect of IL-17, we quantitatively measured the relative expression of T-bet, Eomes, and IFN- $\gamma$ in isolated naïve splenic CD8 T cells that were stimulated in vitro with anti-CD3 and anti-CD28 antibodies in the presence or absence of recombinant IL-17. Not only did the presence of recombinant IL-17 inhibit the expression of both the T-bet and Eomes transcription factors, it also completely abolished the expression of IFN- $\gamma$ in the in vitro cultures (Figure 5J). 
A

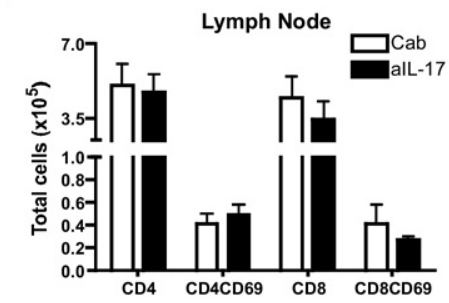

B

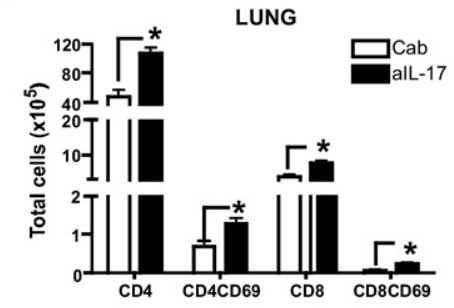

D
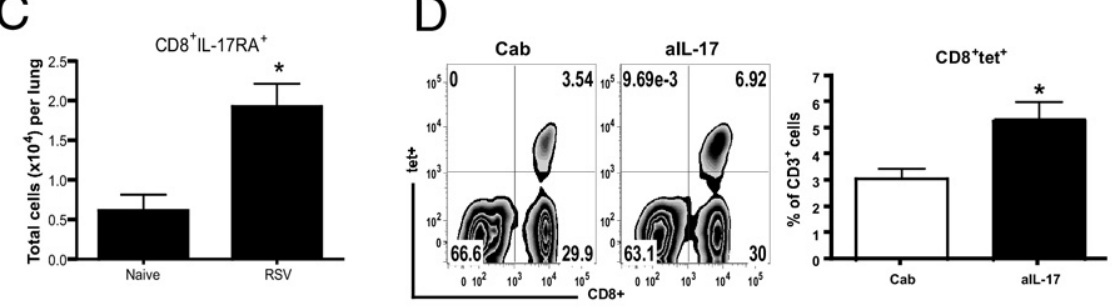

$\mathrm{E}$

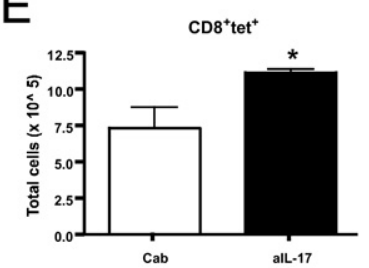

$\mathrm{H}$

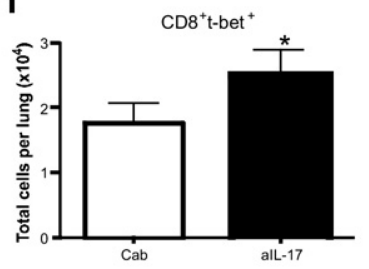

$\mathrm{F}$

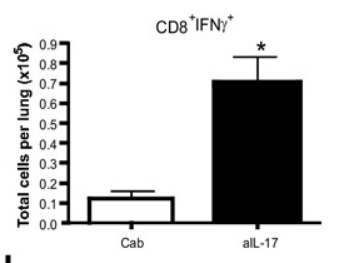

I

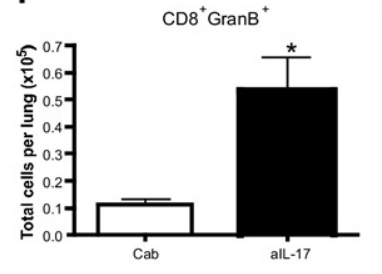

G

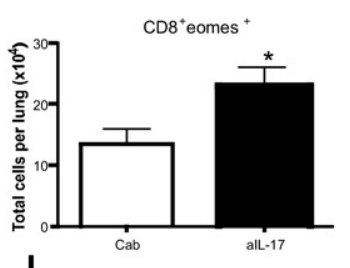
$\mathrm{J}$

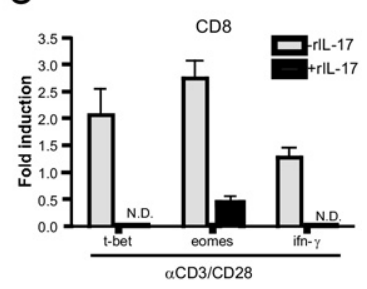

Figure 5. IL-17 inhibits CD $8 \mathrm{~T}$ cell effector responses during RSV infection. Flow cytometry in RSV-infected mice treated with Cab or with antiIL-17 antibody was performed at 8 days after infection using five dispersed lung DLNs (A) or whole-lung digests (B) The results from one experiment of three repeats are shown with total number of CD4 $\left({ }^{*} P<0.04\right)$, CD 4 CD 69 ( ${ }^{*} P<$ $0.02), \mathrm{CD} 8\left({ }^{*} P<0.05\right)$, and CD8CD69 T cells $\left({ }^{*} P<0.05\right)$. C: The number of CD8 T cells positive for IL-17 receptor A chain (IL-17RA) in naïve or RSV-infected mice. D: RSV-specific immunodominant T-cell receptor tetramer surface staining (left), expressed as a percentage of $\mathrm{CD}^{+}$ cells in the lungs (right). E-I: Total tetramerpositive cells (E) IFN- $\gamma(\mathbf{F})$, Eomes (G), T-bet $(\mathbf{H})$, and granzyme B (I) CD8 T cells in the lungs of RSV-infected mice $\left({ }^{*} P<0.05\right)$. J: Relative expression of T-bet, Eomes, and IFN- $\gamma$ was analyzed by real-time PCR in magnetic-cell-sortingpurified CD8 $\mathrm{T}$ cells from the spleen of naive $\mathrm{BALB} / \mathrm{c}$ mice that were stimulated with anti-CD3 $(2 \mu \mathrm{g} / \mathrm{mL})$ and anti-CD28 $(2 \mu \mathrm{g} / \mathrm{mL})$ in the presence $(+$ rIL-17) or absence $(-$ rIL-17) of recombinant IL-17 for 6 hours in vitro. The data were normalized to GAPDH expression levels and the fold induction was compared with unstimulated CD8 T cell values. N.D. indicates not detected.

\section{IL-17 Increases Mucus Production, Alters CD8 T-Cell Activation, and Inhibits Viral Clearance during RSV-Exacerbated Allergic Lung Disease}

Respiratory viral infections are known to exacerbate asthma. Given the results this far in the study, we were interested in whether RSV-induced IL-17 production was involved in exacerbation of our previously developed model of allergic asthma exacerbation. ${ }^{41}$ In this model, BALB/c mice sensitized with CRA are given an RSV infection challenge before a final allergen challenge (Figure 6A). To determine whether RSV increased IL-17 production during allergen challenges in the lungs of mice, lung DLNs from allergen-sensitized mice were isolated and challenged with either allergen or RSV. IL-17 was not detected in cells from any of the three groups of sensitized animals after CRA restimulation (Figure 6B). In contrast, cells from RSV-infected mice restimulated with RSV produced significant amounts of IL-17 (Figure 6B). An even greater and significant increase in IL-17 expression was observed in RSV restimulated cells from mice that were originally sensitized with allergen and then infected with RSV (Figure 6B). These data indicate that RSV infec- tion increases the amount of IL-17 induced in this allergic, Th2 immune environment.

To better understand the contribution of $\mathrm{IL}-17$ in this exacerbation model response, we again used IL-17 neutralization. On day 16, 2 hours before RSV infection, the mice were treated with control or neutralizing antibody to IL-17. The antibodies were administered again on days 18 and 20 and, after a final challenge of CRA on day 21, AHR was measured on day 22. We observed no difference in AHR between control mice and anti-IL-17 antibody-treated mice, indicating that IL-17 does not modulate AHR during exacerbated responses. However, neutralization of IL-17 significantly inhibited mucus production, compared with control mice, as observed from PAS-stained lung sections (Figure 6C). The decrease in mucus production was consistent with a significant decrease in Muc5ac and Gob5 gene expression in the lungs (Figure 6D).

Next, we wanted to determine whether IL-17 mediated regulation of effector CD4 T cell cytokine responses in the lymph nodes. RSV exacerbation of CRA-sensitized mice significantly increased IL-17 production (Figure 6B), and neutralization of IL-17 significantly reduced IL-13 levels, 
A
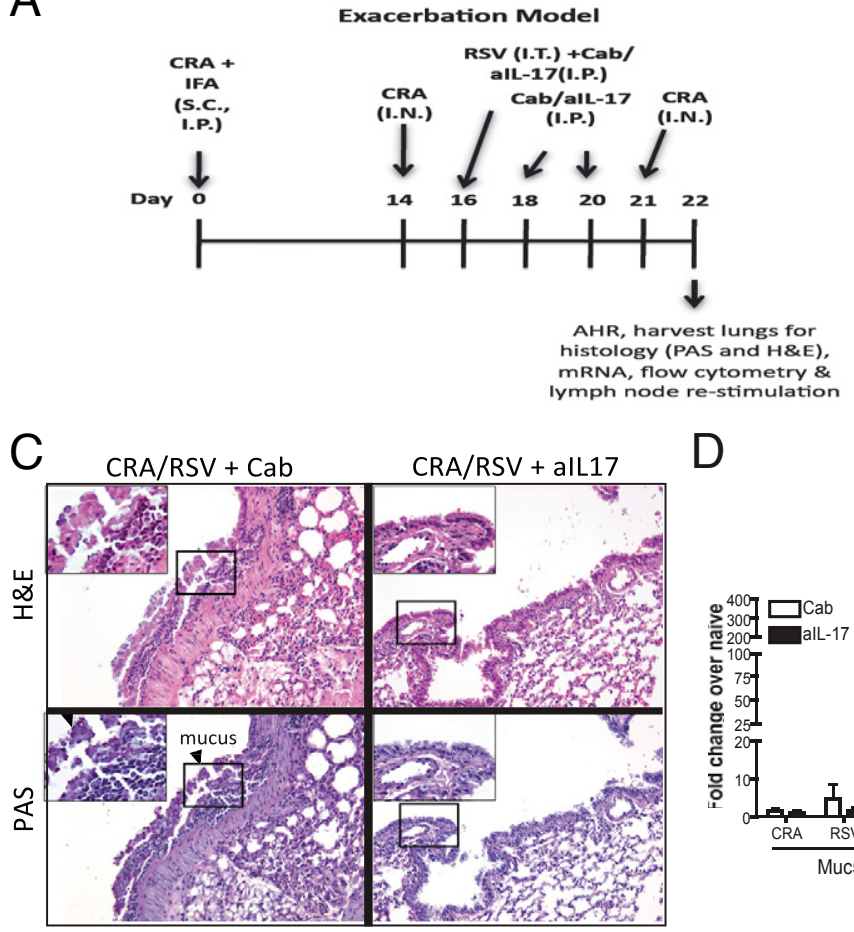

$\mathrm{F}$

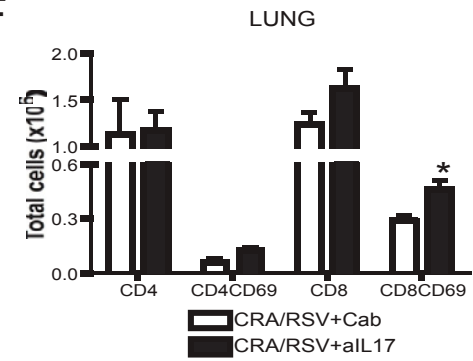

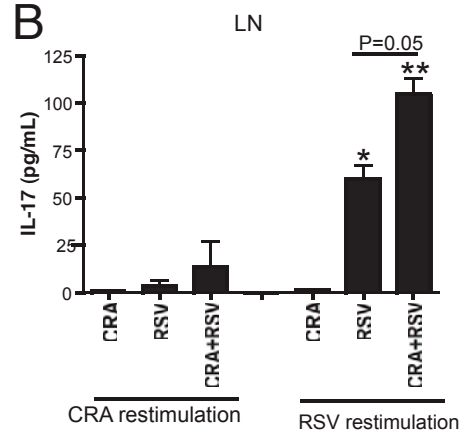

E
LUNG

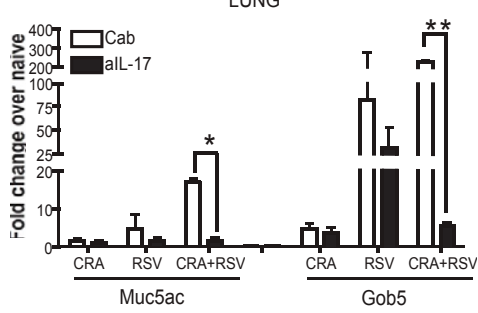

G

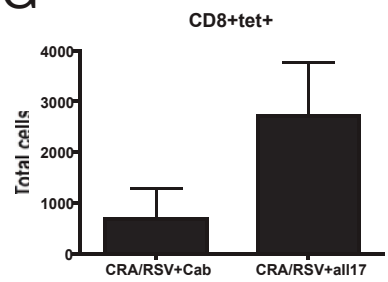

LN

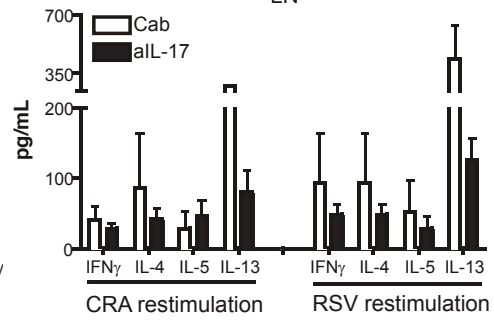

$\mathrm{H}$

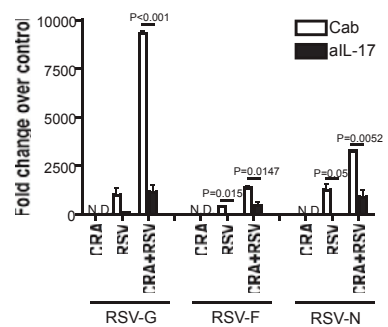

Figure 6. RSV exacerbation of allergic airway disease is mediated by IL-17 by altering mucus production, activating CD8 T cell effector functions, and decreasing viral clearance. A: Schematic representation of RSV exacerbation of existing CRA-induced allergic airway disease. B: Lung DLN cells were isolated from animals sensitized to CRA, RSV, or both CRA and RSV and then were restimulated with either CRA or RSV for 48 hours. The supernatants were analyzed for IL-17 by a Bio-Plex system. C: Lungs from CRA sensitized, RSV-infected mice treated with Cab or anti-IL-17 antibody with H\&E or PAS staining. Original magnification, $\times 400$. Insets: Higher-magnification view of boxed area. Original magnification, $\times 1000$. D: Relative expression of mucus-associated genes (Muc5ac and Gob5) in the lungs was analyzed by real-time PCR. ${ }^{*} P<0.05$, ${ }^{* * *} P<0.01$. E: IFN- $\gamma$, IL-4, IL-5, and IL-13 production was analyzed by a Bio-Plex system. F: Flow cytometry was performed using whole-lung digests. Data are reported as total number of CD4, CD4CD69, CD8, and CD8CD69 T cells per lobe of the lung. * $P<0.05$. G: RSV-specific immunodominant T-cell receptor tetramer surface staining and analysis by flow cytometry. H: Relative expression of RSV proteins (G, F, and N) in the lungs was analyzed by real-time PCR. Data are reported as means \pm SE of five mice per group, representative of one of the two independent experiments. N.D. indicates not detected.

both in response to the individual allergen and to RSV restimulation (Figure 6E) in lymph node cell cultures. These data suggest that in a Th2-skewed environment the RSV-induced IL-17 augments Th2 cytokine production. When $T$ cell accumulation was examined in the lungs of RSV exacerbated animals, a significant increase in CD8CD69 T cells was observed, with no change in CD4 $T$ cell numbers (Figure 6F). Furthermore, there was a marked increase (more than threefold) in RSV antigenspecific CD8 T cells in the lungs of mice treated with neutralizing antibody for IL-17, compared with the Cabtreated mice (Figure 6G). Finally, in mice treated with the anti-IL-17 antibody, a beneficial antiviral response was observed, with a significant decrease in expression of RSV proteins (RSV-G, RSV-F, and RSV-N) (Figure 6H).
Thus, these data confirm that IL-17 directly decreases the antigen-specific CD8 T cells in the lung, increases viral load, and causes mucus production, a combination of effects that suggest a pathogenic role of $\mathrm{IL}-17$ during viral exacerbation of allergic asthma.

\section{Discussion}

Severe RSV infection in infants is characterized by mucus plugging of the airways, airway hyperreactivity, and inflammation. ${ }^{42,43}$ Although an association with Th2 cytokines has been established with severe RSV disease, ${ }^{44}$ less is known about the role of IL-17. In the present study, using a mouse model of RSV infection, 
we investigated the role of IL-17 during primary RSV infection. We found that IL-17 modulates three important underlying mechanisms: i) hypermucus production in the airways, ii) alteration of effector CD8 T cell responses, and iii) viral clearance. Each of these mechanisms may contribute to RSV-induced pulmonary pathology. In addition, IL-17 produced during RSV infection appears to participate in RSV-induced exacerbation of allergic lung disease, as previously suggested. ${ }^{15}$ The relevance of these observations was supported by the detection of IL-17 in tracheal samples from severely ill infants infected by RSV.

The ability of IL-17 produced during RSV infection to regulate mucus production was identified by neutralization of IL-17. Given that IL-17 neutralization had no effect on AHR, it may be that other cytokines (especially Th2 cytokines) influence the airway function: whereas IL-17 alters the epithelial cell response that controls mucus production. This pathogenic effect of IL-17 on mucus production may be important in several diseases, including chronic obstructive pulmonary disease, cystic fibrosis, and asthma. IL-17 did not alter the CD4 effector responses and Th2 cytokine production profile during RSV infection, suggesting that IL-17 may be functioning primarily as an effector cytokine leading to increased mucus production.

Of note, when we assessed Th2 cytokine production in restimulated lymph node cells from RSV-exacerbated allergic mice, the neutralization of IL-17 significantly reduced IL-13 levels. Thus, the role of IL-17 in a Th2skewed environment appears to enhance Th2 cytokine production. The latter observation is consistent with the reduced expression of Th2 cytokines observed in IL-17 knockout mice in the ovalbumin-alum-induced asthma model. ${ }^{12,13}$ There are differences between IL-17 and IL$17 \mathrm{~F}$ in regulating Th2 responses. Whereas IL-17 appears to promote Th2 responses, IL-17F has a regulatory role in restricting Th2 responses. ${ }^{45}$ We speculate that the relative lower expression of IL-17F during RSV infection may contribute to the heightened IL-13 response during an allergic condition; however, further experimental evidence is required. More recently, the $\mathrm{IL}-13$ receptor was found on Th17 cells and was shown to regulate IL-17 production. ${ }^{46}$ The relationship of IL-17 and IL-13 in regulating mucus production during RSV exacerbated disease still remains to be established, but may demonstrate that the two cytokines play synergistic roles in mucus regulation. This would be particularly important during disease exacerbation in allergic asthma, in which virusinduced IL-17 in a Th2 immune environment may present the most severe disease phenotype.

$\mathrm{IL}-17$ has also been associated with increased neutrophilic infiltration at the site of infection during host responses to bacterial and fungal infections through induction of CXC chemokines. ${ }^{47,48}$ Current evidence indicates that viral infection of epithelial cells increases the production of neutrophil chemoattractants, which induce neutrophil migration into the inflammatory sites. ${ }^{49-51}$ Consistent with our present findings, it was reported that IL-17 enhances human rhinovirus-16-induced epithelial production of IL-8 (human homolog of mouse KC) ${ }^{52}$ Neutrophils augment epithelial cell damage by the release of granule enzymes during viral infection and thus contribute to the pathophysiology of viral disease. ${ }^{53}$ Allergic disease may therefore present the most severe phenotype when intense neutrophil recruitment to the lung occurs, as has been observed in clinical disease. ${ }^{54,55}$

CD8 T cell antiviral responses confer protective immunity during viral infections. Recently, it was reported that IL-17 impaired CD8 T cell antiviral responses during Theiler's murine encephalomyelitis virus (TMEV) infections, ${ }^{56}$ and IL-17 was also shown to inhibit CD8 T cell cytotoxic function against tumor cells. ${ }^{57}$ Accordingly, an aspect of the regulation of CD8 T cell response in the present study was linked to CD4 T cell help, given that CD4 T cells were the primary source of IL-17 during RSV infection. Two T-box-containing transcription factors, T-bet and Eomes, regulate the CD8 cytotoxic $T$ cell effector functions. ${ }^{58}$ Consequently, $T$ cells lacking both T-bet and Eomes fail to control lymphocytic choriomeningitis virus (LCMV) infection..$^{59}$ Of note, an aberrant Th17 response developed in studies with mice that were deficient in both Eomes and T-bet, suggesting that there may be an antagonistic relationship between the expression of these molecules. ${ }^{59}$ We observed negative regulation of T-bet and Eomes by IL-17, indicating an inhibitory role for IL-17 in cytotoxic CD8 T cell responses. Furthermore, in the presence of $\mathrm{IL}-17$ using in vitro cultures, expression levels of IFN- $\gamma$, Eomes, and T-bet in CD8 T cells were significantly decreased, suggesting that IL-17 directly regulates CTL development. Thus, the negative regulatory effects of IL-17 on cytotoxic CD8 T cells during both RSV infection and the exacerbated allergic response contribute to the viral associated pathology.

In the present study, we found that virus-induced IL-17 augments primary RSV infection, as well as existing airway disease, by increasing mucus production, inhibiting CD8 T cell activation, and reducing viral clearance. Conversely, removing IL-17 led to a reduction of the exacerbated disease, possibly in part by increasing viral clearance. Our observation of increased IL-17 in airway samples of severely ill RSV-infected infants and the role of $\mathrm{IL}-17$ in mucus hypersecretion can be implicated in chronic lung conditions such as chronic obstructive pulmonary disease and asthma during exacerbation by RSV. ${ }^{60-63}$

\section{Acknowledgments}

We thank Drs. Matthew Schaller and Lara K. Kallal for helpful discussions and Dr. Judith Connett for her careful reading of the manuscript.

\section{References}

1. Openshaw PJ, Dean GS, Culley FJ: Links between respiratory syncytial virus bronchiolitis and childhood asthma: clinical and research approaches. Pediatr Infect Dis J 2003, 22(2 Suppl):S58-S64; discussion S64-S65

2. Black CP: Systematic review of the biology and medical management of respiratory syncytial virus infection. Respir Care 2003, 48:209-231; discussion 231-233 
3. Stensballe LG, Devasundaram JK, Simoes EA: Respiratory syncytial virus epidemics: the ups and downs of a seasonal virus. Pediatr Infect Dis J 2003, 22(2 Suppl):S21-S32

4. Jafri HS, Chavez-Bueno S, Mejias A, Gomez AM, Rios AM, Nassi SS, Yusuf M, Kapur P, Hardy RD, Hatfield J, Rogers BB, Krisher K, Ramilo $\mathrm{O}$ : Respiratory syncytial virus induces pneumonia, cytokine response, airway obstruction, and chronic inflammatory infiltrates associated with long-term airway hyperresponsiveness in mice. J Infect Dis 2004 189:1856-1865

5. Schwarze J, Cieslewicz G, Hamelmann E, Joetham A, Shultz LD, Lamers MC, Gelfand EW: IL-5 and eosinophils are essential for the development of airway hyperresponsiveness following acute respiratory syncytial virus infection. J Immunol 1999, 162:2997-3004

6. Tekkanat KK, Maassab H, Miller A, Berlin AA, Kunkel SL, Lukacs NW: RANTES (CCL5) production during primary respiratory syncytial virus infection exacerbates airway disease. Eur J Immunol 2002, 32:32763284

7. Hashimoto K, Durbin JE, Zhou W, Collins RD, Ho SB, Kolls JK, Dubin PJ, Sheller Jr, Goleniewska K, O'Neal JF, Olson SJ, Mitchell D, Graham BS, Peebles RS Jr: Respiratory syncytial virus infection in the absence of STAT 1 results in airway dysfunction, airway mucus, and augmented IL-17 levels. J Allergy Clin Immunol 2005, 116:550-557

8. Hymowitz SG, Filvaroff EH, Yin JP, Lee J, Cai L, Risser P, Maruoka M, Mao W, Foster J, Kelley RF, Pan G, Gurney AL, de Vos AM, Starovasnik MA: IL-17s adopt a cystine knot fold: structure and activity of a novel cytokine, IL-17F, and implications for receptor binding. EMBO J 2001, 20:5332-5341

9. Kawaguchi M, Onuchic LF, Li XD, Essayan DM, Schroeder J, Xiao $H Q$, Liu MC, Krishnaswamy G, Germino G, Huang SK: Identification of a novel cytokine, ML-1, and its expression in subjects with asthma. $\mathrm{J}$ Immunol 2001, 167:4430-4435

10. Hizawa N, Kawaguchi M, Huang SK, Nishimura M: Role of interleukin$17 \mathrm{~F}$ in chronic inflammatory and allergic lung disease. Clin Exp Allergy 2006, 36:1109-1114

11. Ouyang W, Kolls JK, Zheng Y: The biological functions of T helper 17 cell effector cytokines in inflammation. Immunity 2008, 28:454-467

12. Yang $X O$, Chang SH, Park $H$, Nurieva R, Shah B, Acero L, Wang YH, Schluns KS, Broaddus RR, Zhu Z, Dong C: Regulation of inflammatory responses by IL-17F. J Exp Med 2008, 205:1063-1075

13. Nakae S, Komiyama $Y$, Nambu A, Sudo K, Iwase M, Homma I, Sekikawa K, Asano M, Iwakura Y: Antigen-specific T cell sensitization is impaired in IL-17-deficient mice, causing suppression of allergic cellular and humoral responses. Immunity 2002, 17:375-387

14. Schnyder-Candrian S, Togbe D, Couillin I, Mercier I, Brombacher F, Quesniaux V, Fossiez F, Ryffel B, Schnyder B: Interleukin-17 is a negative regulator of established allergic asthma. J Exp Med 2006 , 203:2715-2725

15. Hashimoto K, Graham BS, Ho SB, Adler KB, Collins RD, Olson SJ, Zhou W, Suzutani T, Jones PW, Goleniewska K, O'Neal JF, Peebles RS Jr: Respiratory syncytial virus in allergic lung inflammation increases Muc5ac and gob-5. Am J Respir Crit Care Med 2004, 170: 306-312

16. Tekkanat KK, Maassab H, Berlin AA, Lincoln PM, Evanoff HL, Kaplan $\mathrm{MH}$, Lukacs NW: Role of interleukin-12 and stat-4 in the regulation of airway inflammation and hyperreactivity in respiratory syncytial virus infection. Am J Pathol 2001, 159:631-638

17. Al-Ramli W, Préfontaine D, Chouiali F, Martin JG, Olivenstein R, Lemiere C, Hamid Q: T(H)17-associated cytokines (IL-17A and IL17F) in severe asthma. J Allergy Clin Immunol 2009, 123:1185-1187

18. Yang $X O$, Nurieva R, Martinez GJ, Kang HS, Chung Y, Pappu BP, Shah B, Chang SH, Schluns KS, Watowich SS, Feng XH, Jetten AM, Dong C: Molecular antagonism and plasticity of regulatory and inflammatory T cell programs. Immunity 2008, 29:44-56

19. Campbell EM, Charo IF, Kunkel SL, Strieter RM, Boring L, Gosling J, Lukacs NW: Monocyte chemoattractant protein-1 mediates cockroach allergen-induced bronchial hyperreactivity in normal but not CCR2-/- mice: the role of mast cells. J Immunol 1999, 163:2160-2167

20. Campbell EM, Kunkel SL, Strieter RM, Lukacs NW: Temporal role of chemokines in a murine model of cockroach allergen-induced airway hyperreactivity and eosinophilia. J Immunol 1998, 161:7047-7053

21. Moore ML, Chi MH, Luongo C, Lukacs NW, Polosukhin VV, Huckabee MM, Newcomb DC, Buchholz UJ, Crowe JE Jr, Goleniewska K, Williams JV, Collins PL, Peebles RS Jr: A chimeric A2 strain respiratory syncytial virus (RSV) with the fusion protein of RSV strain line 19 exhibits enhanced viral load, mucus, and airway dysfunction. J Virol 2009, 83:4185-4194

22. Herlocher ML, Ewasyshyn M, Sambhara S, Gharaee-Kermani M, Cho D, Lai J, Klein M, Maassab HF: Immunological properties of plaque purified strains of live attenuated respiratory syncytial virus (RSV) for human vaccine. Vaccine 1999, 17:172-181

23. Lukacs NW, Moore ML, Rudd BD, Berlin AA, Collins RD, Olson SJ, Ho SB, Peebles RS Jr: Differential immune responses and pulmonary pathophysiology are induced by two different strains of respiratory syncytial virus. Am J Pathol 2006, 169:977-986

24. Rudd BD, Smit JJ, Flavell RA, Alexopoulou L, Schaller MA, Gruber A Berlin AA, Lukacs NW: Deletion of TLR3 alters the pulmonary immune environment and mucus production during respiratory syncytial virus infection. J Immunol 2006, 176:1937-1942

25. Smit JJ, Rudd BD, Lukacs NW: Plasmacytoid dendritic cells inhibit pulmonary immunopathology and promote clearance of respiratory syncytial virus. J Exp Med 2006, 203:1153-1159

26. Miller AL, Gerard C, Schaller M, Gruber AD, Humbles AA, Lukacs NW: Deletion of CCR1 attenuates pathophysiologic responses during respiratory syncytial virus infection. J Immunol 2006, 176:2562-2567

27. Miller AL, Bowlin TL, Lukacs NW: Respiratory syncytial virus-induced chemokine production: linking viral replication to chemokine production in vitro and in vivo. J Infect Dis 2004, 189:1419-1430

28. John AE, Gerard CJ, Schaller M, Miller AL, Berlin AA, Humbles AA, Lukacs NW: Respiratory syncytial virus-induced exaggeration of allergic airway disease is dependent upon CCR1-associated immune responses [Erratum appeared in Eur J Immunol 2005;35:3083]. Eur $\mathrm{J}$ Immunol 2005, 35:108-116

29. Ito T, Schaller M, Hogaboam CM, Standiford TJ, Sandor M, Lukacs NW, Chensue SW, Kunkel SL: TLR9 regulates the mycobacteriaelicited pulmonary granulomatous immune response in mice through DC-derived Notch ligand delta-like 4. J Clin Invest 2009, 119:33-46

30. Kallal LE, Hartigan AJ, Hogaboam CM, Schaller MA, Lukacs NW: Inefficient lymph node sensitization during respiratory viral infection promotes IL-17-mediated lung pathology. J Immunol 2010, 185: 4137-4147

31. Lukacs NW, Smit JJ, Mukherjee S, Morris SB, Nunez G, Lindell DM: Respiratory virus-induced TLR7 activation controls IL-17-associated increased mucus via IL-23 regulation. J Immunol 2010, 185:22312239

32. Hogaboam CM, Gallinat CS, Taub DD, Strieter RM, Kunkel SL, Lukacs NW: Immunomodulatory role of C10 chemokine in a murine model of allergic bronchopulmonary aspergillosis. J Immunol 1999, 162:6071-6079

33. Lukacs NW, Tekkanat KK, Berlin A, Hogaboam CM, Miller A, Evanoff $\mathrm{H}$, Lincoln P, Maassab H: Respiratory syncytial virus predisposes mice to augmented allergic airway responses via IL-13-mediated mechanisms [Erratum appeared in J Immunol 2005;175:8442]. J Immunol 2001, 167:1060-1065

34. Tekkanat KK, Maassab HF, Cho DS, Lai JJ, John A, Berlin A, Kaplan $\mathrm{MH}$, Lukacs NW: IL-13-induced airway hyperreactivity during respiratory syncytial virus infection is STAT6 dependent [Erratum appeared in J Immunol 2005;175:8442]. J Immunol 2001, 166:35423548

35. Kumar M, Behera AK, Matsuse H, Lockey RF, Mohapatra SS: Intranasal IFN-gamma gene transfer protects BALB/c mice against respiratory syncytial virus infection. Vaccine 1999, 18:558-567

36. Matsuse H, Behera AK, Kumar M, Lockey RF, Mohapatra SS: Differential cytokine mRNA expression in Dermatophagoides farinae allergen-sensitized and respiratory syncytial virus-infected mice. Microbes Infect 2000, 2:753-759

37. Lundy SK, Lira SA, Smit JJ, Cook DN, Berlin AA, Lukacs NW: Attenuation of allergen-induced responses in CCR6-/- mice is dependent upon altered pulmonary T lymphocyte activation. J Immunol 2005, 174:2054-2060

38. Medzhitov R: Recognition of microorganisms and activation of the immune response. Nature 2007, 449:819-826

39. Wang SZ, Forsyth KD: The interaction of neutrophils with respiratory epithelial cells in viral infection. Respirology 2000, 5:1-10

40. Weaver CT, Hatton RD, Mangan PR, Harrington LE: IL-17 family cytokines and the expanding diversity of effector $T$ cell lineages. Annu Rev Immunol 2007, 25:821-852 
41. Schaller MA, Kallal LE, Lukacs NW: A key role for CC chemokine receptor 1 in T-cell-mediated respiratory inflammation. Am J Pathol 2008, 172:386-394

42. Welliver RC: Review of epidemiology and clinical risk factors for severe respiratory syncytial virus (RSV) infection. J Pediatr 2003, 143(5 Suppl):S112-S117

43. Martinez FD: Respiratory syncytial virus bronchiolitis and the pathogenesis of childhood asthma. Pediatr Infect Dis J 2003, 22(2 Suppl): S76-S82

44. Tripp RA, Oshansky C, Alvarez R: Cytokines and respiratory syncytial virus infection. Proc Am Thorac Soc 2005, 2:147-149

45. Chang SH, Dong C: IL-17F: regulation, signaling and function in inflammation. Cytokine 2009, 46:7-11

46. Newcomb DC, Zhou W, Moore ML, Goleniewska K, Hershey GK, Kolls JK, Peebles RS Jr: A functional IL-13 receptor is expressed on polarized murine CD4+ Th17 cells and IL-13 signaling attenuates Th17 cytokine production. J Immunol 2009, 182:5317-5321

47. Ye P, Rodriguez FH, Kanaly S, Stocking KL, Schurr J, Schwarzenberger P, Oliver P, Huang W, Zhang P, Zhang J, Shellito JE, Bagby GJ, Nelson S, Charrier K, Peschon JJ, Kolls JK: Requirement of interleukin 17 receptor signaling for lung CXC chemokine and granulocyte colony-stimulating factor expression, neutrophil recruitment, and host defense. J Exp Med 2001, 194:519-527

48. Osorio F, LeibundGut-Landmann S, Lochner M, Lahl K, Sparwasser T, Eberl G, Reis e Sousa C: DC activated via dectin-1 convert Treg into IL-17 producers. Eur J Immunol 2008, 38:3274-3281

49. Chintakuntlawar AV, Chodosh J: Chemokine CXCL1/KC and its receptor CXCR2 are responsible for neutrophil chemotaxis in adenoviral keratitis. J Interferon Cytokine Res 2009, 29:657-666

50. Vieira SM, Lemos HP, Grespan R, Napimoga MH, Dal-Secco D Freitas A, Cunha TM, Verri WA Jr, Souza-Junior DA, Jamur MC, Fernandes KS, Oliver C, Silva JS, Teixeira MM, Cunha FQ: A crucial role for TNF-alpha in mediating neutrophil influx induced by endogenously generated or exogenous chemokines, KC/CXCL1 and LIX/ CXCL5. Br J Pharmacol 2009, 158:779-789

51. Crowe CR, Chen K, Pociask DA, Alcorn JF, Krivich C, Enelow RI, Ross TM, Witztum JL, Kolls JK: Critical role of IL-17RA in immunopathology of influenza infection. J Immunol 2009, 183:5301-5310
52. Wiehler S, Proud D: Interleukin-17A modulates human airway epithelial responses to human rhinovirus infection. Am J Physiol Lung Cell Mol Physiol 2007, 293:L505-L515

53. Jaovisidha P, Peeples ME, Brees AA, Carpenter LR, Moy JN: Respiratory syncytial virus stimulates neutrophil degranulation and chemokine release. J Immunol 1999, 163:2816-2820

54. Dougherty RH, Fahy JV: Acute exacerbations of asthma: epidemiology, biology and the exacerbation-prone phenotype. Clin Exp Allergy 2009, 39:193-202

55. Wark PA, Johnston SL, Moric I, Simpson JL, Hensley MJ, Gibson PG: Neutrophil degranulation and cell lysis is associated with clinical severity in virus-induced asthma. Eur Respir J 2002, 19:68-75

56. Hou W, Kang HS, Kim BS: Th17 cells enhance viral persistence and inhibit $\mathrm{T}$ cell cytotoxicity in a model of chronic virus infection. J Exp Med 2009, 206:313-328

57. Numasaki M, Fukushi J, Ono M, Narula SK, Zavodny PJ, Kudo T, Robbins PD, Tahara H, Lotze MT: Interleukin-17 promotes angiogenesis and tumor growth. Blood 2003, 101:2620-2627

58. Pearce EL, Mullen AC, Martins GA, Krawczyk CM, Hutchins AS, Zediak VP, Banica M, DiCioccio CB, Gross DA, Mao CA, Shen H, Cereb N, Yang SY, Lindsten T, Rossant J, Hunter CA, Reiner SL: Control of effector CD8+ T cell function by the transcription factor eomesodermin. Science 2003, 302:1041-1043

59. Intlekofer AM, Banerjee A, Takemoto N, Gordon SM, Dejong CS, Shin H, Hunter CA, Wherry EJ, Lindsten T, Reiner SL: Anomalous type 17 response to viral infection by CD8 $+\mathrm{T}$ cells lacking $\mathrm{T}$-bet and eomesodermin. Science 2008, 321:408-411

60. Ramaswamy M, Groskreutz DJ, Look DC: Recognizing the importance of respiratory syncytial virus in chronic obstructive pulmonary disease. COPD 2009, 6:64-75

61. Johnston NW: The similarities and differences of epidemic cycles of chronic obstructive pulmonary disease and asthma exacerbations. Proc Am Thorac Soc 2007, 4:591-596

62. Falsey AR: Respiratory syncytial virus infection in adults. Semin Respir Crit Care Med 2007, 28:171-181

63. Welliver RC: RSV and chronic asthma. Lancet 1995, 346:789-790 